\title{
Method of Experimentally Identifying the Complex Mode Shape of the Self-Excited Oscillation of a Cantilevered Pipe Conveying Fluid
}

Eisuke Higuchi ( $\sim$ s2120762@s.tsukuba.ac.jp )

University of Tsukuba: Tsukuba Daigaku https://orcid.org/0000-0002-7172-9056

Hiroshi Yabuno

University of Tsukuba: Tsukuba Daigaku

Kiyotaka Yamashita

Fukui University of Technology: Fukui Kogyo Daigaku

\section{Research Article}

Keywords: Pipe conveying fluid, Complex mode, Experiment

Posted Date: December 6th, 2021

DOl: https://doi.org/10.21203/rs.3.rs-922532/v1

License: (c) (i) This work is licensed under a Creative Commons Attribution 4.0 International License.

Read Full License 


\title{
Method of experimentally identifying the complex mode shape of the self-excited oscillation of a cantilevered pipe conveying fluid
}

\author{
Eisuke Higuchi · Hiroshi Yabuno - Kiyotaka Yamashita
}

Received: date / Accepted: date

\begin{abstract}
The dynamics of a flexible cantilevered pipe conveying fluid have been researched for several decades. It is known that the flexible pipe undergoes self-excited vibration when the flow speed exceeds a critical speed. This instability phenomenon is caused by nonconservative forces. From a mathematical point of view, the system has a characteristic of non-selfadjointness and the linear eigenmodes can be complex and non-orthogonal to each other. As a result, such a mathematical feature of the system is directly related to the instability phenomenon. In this study, we propose a method of experimentally identifying the complex mode from experimentally obtained time histories and decomposing the linear mode into real and imaginary components. In nonlinear analysis, we show that the nonlinear effects of practical systems on the mode in the steady-state selfexcited oscillation are small. The real and imaginary components identified using the proposed method for experimental steady-state self-excited oscillations are compared with those obtained in the theoretical analysis, thus validating the proposed identification method.
\end{abstract}

Keywords Pipe conveying fluid . Complex mode . Experiment

\footnotetext{
E. Higuchi

1-1-1, Tennodai, Tsukuba City, Ibaraki Prefecture, Japan

Tel.: +81 029-853-6470

Fax: +81 029-853-6470

E-mail: s2120762@s.tsukuba.ac.jp

H. Yabuno

1-1-1, Tennodai, Tsukuba City, Ibaraki Prefecture, Japan

K. Yamashita

3-6-1, Gakuen, Fukui City, Fukui Prefecture, Japan
}

\section{Introduction}

Instability phenomena of a flexible pipe conveying fluid have been investigated for several decades. It is known that the dynamics of a pipe depend on the support condition. A simply supported pipe loses stability through divergence [6] whereas a cantilevered pipe is dynamically destabilized by flutter [10]. The latter phenomenon results from a nonconservative follower force, which is the fluid force of the internal flow acting on the pipe orthogonally to the cross section of the pipe [21]. It is well known that when the flow speed of the fluid in the pipe exceeds a critical speed, the trivial steady-state amplitude becomes unstable and Hopf bifurcation occurs [3]. After the transient state, the pipe exhibits steadystate self-excited oscillation with a constant amplitude. From a mathematical point of view, the nonconservative force makes the system non-selfadjoint [13] and the eigenmodes can then be complex and non-orthogonal to each other.

The first serious study on the dynamics of a pipe conveying fluid was Bourrieres's work in 1939, but it was lost [18]. After this work, Ashley and Haviland [1] investigated vibrations of the oil pipeline. A pioneering theoretical study of the dynamic destabilization phenomenon due to such fluid-solid interaction was conducted using an articulated pipe by Benjamin [4]. Gregory and Païdoussis [8] conducted a theoretical analysis of continuous pipes with an infinite number of degrees of freedom. Additionally, the relationship between system parameters and stability was investigated by Bishop and Fawzy [5]. After these studies, many associated studies $[18,19]$ were performed owing to the characteristic excitation mechanism and the specific mathematical features that the system can have non-selfadjointness and non-orthogonal and complex eigenmodes. For ex- 
ample, there have been studies of the effects of pulsating internal fluid on motion and stability $[11,24,30]$. Early works, such as $[4,5,8]$, focused on the planar motion of the pipe. Lundgren et al. and Bajaj and Sethna conducted a theoretical analysis of the non-planar motion of a plain pipe $[2,14]$. Following these works, the non-planar motions of pipes with an end mass [15] and spring support $[7,22,23]$ were examined.

Most of the studies mentioned above investigated in detail the change in the eigenvalues, which relate to the stability of the system directly, with respect to changes in system parameters, but there has been less consideration of the complex eigenmode due to nonselfadjointness $[27,29]$ and no research from an experimental point of view to the best of our knowledge. The pioneering work of Benjamin [4] noted the characteristics of the mode shape but only qualitatively, and there has been no investigation of the mode shape since.

Incidentally, in most theoretical studies, the Galerkin discretization scheme has been used to examine the equation of the motion of a flexible pipe conveying fluid $[8,12,20]$. In this analytical approach, the modes are assumed by the modes of the free-oscillation in which the effect of the internal flow is not taken into account. This assumption neglects the non-selfadjointness of the system, that the eigenmodes can be complex and the nonorthogonality of the modes. These mathematical characteristics result from nonconservative forces directly and must therefore be considered in not only theoretical investigations but also experimental studies. It is thus necessary to express the vibration mode using a complex mode and to capture the mode shape through the superposition of its real and imaginary components. This approach will provide a deep understanding of the characteristics of the instability phenomena of non-selfadjoint systems.

Experimental studies on the mode shape of instability vibration have been limited to observing stroboscopic photographs of the mode shape, as done by Gregory and Païdoussis [9] and Yoshizawa [29]. We cannot capture the complex mode from these photographs. Unlike the case when the eigenmodes are real, when the eigenmodes are complex, the phases of vibration at each point on the pipe are expressed as a function of time and position, and different points on the pipe thus have different phases. This characteristic of the mode is thought to produce characteristic vibration shapes such as the "dragging" shape noted by [4,9]. Such a feature of the mode cannot presently be detected using conventional methods such as stroboscopic photography. A new method of obtaining a complex mode and expressing the real and imaginary components experimentally is needed.
In this study, first, using linear theory, we obtain the complex-valued eigenmode theoretically considering the non-selfadjointness of the system. The eigenmode is shown as the composition of the real and imaginary components. We then clarify that the phase of the lateral vibration at each point on the pipe is a function of the distance from the supporting point. Next, nonlinear analysis shows that the nonlinear elastic and inertia forces play an important role in determining the magnitude of the amplitude in steady-state self -excited oscillation, but do not have a small effect on the eigenmode.

Second, using this knowledge, we present an experimental identification method for detecting the real and imaginary components of an imaginary eigenmode shape of self-excited vibration from experimentally obtained data of the time histories of some points. In practice, using the time history data of steady-state selfexcited oscillation obtained for a cantilevered pipe conveying fluid in our experimental apparatus, we experimentally identify the complex eigenmode and decomposed the real and imaginary components. The comparison of the components with those obtained theoretically demonstrates the validity of the proposed experimental identification method for the complex eigenmode of self-excited oscillation generated by the nonselfadjointness.

\section{Complex linear normal mode due to the non-selfadjointness}

2.1 Governing equation of a cantilevered elastic pipe conveying fluid

We consider the analytical model for the vibration of a cantilevered elastic pipe conveying fluid as shown in Fig. 1. The pipe is hung vertically under the influence of gravity $g$ and the upper end is clamped. The pipe is assumed to be a flexible and inextensible beam with flexural rigidity $E I$. The length, mass per unit length and cross-sectional flow area of the pipe are respectively denoted $l, m$ and $S$. The pipe is sufficiently long compared with its diameter and conveys an incompressible fluid having a mass per unit length $M$. The flow in the pipe is assumed to be constant and one-dimensional, and the flow velocity $U$ relative to the pipe motion is assumed to be parallel to the pipe centerline. Under special conditions, there is non-planar motion $[2,25]$, but to gain a fundamental understanding of the complex eigenmode, we consider the motion in a vertical $x-y$ plane. We introduce the $x-y$ coordinate system, whose origin $O$ is located at the upper end of the pipe. In addition, we 


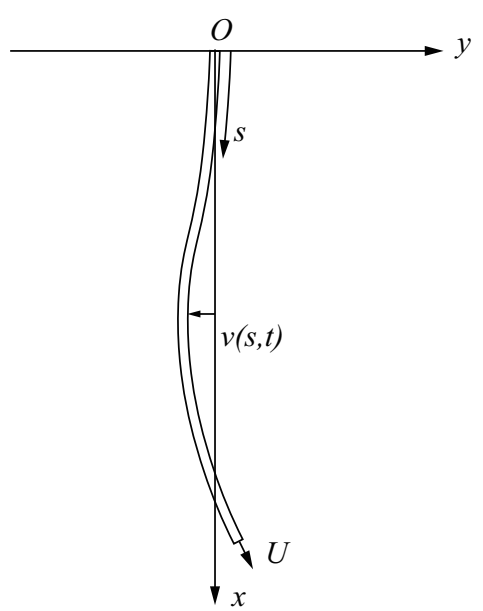

Fig. 1 Analytical model of a cantilevered elastic pipe conveying fluid

let $s$ be the curvilinear coordinate along the centerline of the pipe.

Let $v$ be the displacement along the pipe centerline in the $y$ direction. Using $l$ as the representative length $L$ and $\sqrt{(m+M) L^{4} / E I}$ as the representative time $T$, we introduce the dimensionless parameters $\beta, \gamma$ and $U^{*}$ as

$\beta=\frac{M}{m+M}, \gamma=\frac{(m+M) g l^{3}}{E I}, U^{*}=\sqrt{\frac{M l^{2}}{E I}} U$,

where $\beta, \gamma$ and $U^{*}$ respectively denote the ratio of the fluid mass to the total mass, the ratio of the gravity force to the elastic force of the pipe, and the dimensionless flow velocity. The equation of the pipe conveying fluid can be written with terms up to the third order of $v^{*}$ in non-dimensional form $[17,28]$ as

$v^{*^{\prime \prime \prime \prime}}+U^{2} v^{*^{\prime \prime}}+2 \sqrt{\beta} U \dot{v}^{*^{\prime}}+\gamma\left\{v^{*^{\prime}}-(1-s) v^{*^{\prime \prime}}\right\}+\ddot{v}^{*}$

$-\left\{v^{*^{\prime}} \frac{\partial^{2}}{\partial t^{2}} \int_{0}^{s}\left(-\frac{1}{2} v^{*^{\prime 2}}\right) d s-U \sqrt{\beta} v^{*^{\prime 2}} \dot{v}^{*^{\prime}}+\frac{1}{2} v^{*^{\prime 2}} \ddot{v}^{*}\right.$

$-\frac{1}{2} U^{2} v^{*^{\prime 2}} v^{*^{\prime \prime}}-\left(\frac{3}{2} v^{*^{\prime \prime 3}}+3 v^{*^{\prime}} v^{*^{\prime \prime}} v^{*^{\prime \prime \prime}}+\frac{1}{2} v^{*^{\prime 2}} v^{*^{\prime \prime \prime \prime}}\right)$

$-v^{*^{\prime \prime}} \int_{s}^{1}\left(\frac{\partial^{2}}{\partial t^{2}} \int_{0}^{s}\left(-\frac{1}{2}\right) d s+v^{*^{\prime}} \ddot{v}^{*}\right) d s$

$-\frac{1}{2} \gamma v^{*^{\prime \prime}} \int_{s}^{1} v^{*^{\prime 2}} d s+\frac{1}{2} v^{*^{\prime 2}} v^{*^{\prime \prime}} \gamma(1-s)$

$\left.+\left.\frac{1}{2} v^{*^{\prime \prime 2}}\right|_{s=1} v^{*^{\prime \prime}}\right\}=0$

where (') and (') respectively denote the derivatives with respect to dimensionless time $t$ and position $s$. Hereafter, we omit the $\left(^{*}\right)$ notation for simplicity. On the left-hand side of Eq. (2), the second, third and fourth terms are respectively associated with centrifugal, Coriolis and gravitational forces.
The boundary conditions for the pipe are expressed as

$s=0: v(0)=0, v^{\prime}(0)=0$,

$s=1: v^{\prime \prime}(1)=0, v^{\prime \prime \prime}(1)=0$.

\subsection{Linear modal analysis}

By neglecting the nonlinear terms with respect to $v$ in Eq. (2), we obtain the linear partial differential equation

$v^{\prime \prime \prime \prime}+\left\{U^{2}-\gamma(1-s)\right\} v^{\prime \prime}+2 \sqrt{\beta} U \dot{v^{\prime}}+\gamma v^{\prime}+\ddot{v}=0$

and the associated boundary conditions

$s=0: v(0)=0, v^{\prime}(0)=0$,

$s=1: v^{\prime \prime}(1)=0, v^{\prime \prime \prime}(1)=0$.

The eigenfrequencies and associated eigenmodes, which are obtained from Eqs. (4) and (5), have been well investigated [18] but we summarize the modulations of the eigenfrequencies and eigenmodes depending on the flow velocity because they relate to the main topic of complex eigenmodes. We here express the mode shapes using a power series of the coordinate $s$ [29].

Let $v$ be

$v=\sum_{n=1}^{\infty} A_{n} \Phi_{n}(s) \mathrm{e}^{\lambda_{n} t}+C . C$.

where $A_{n}, \Phi_{n}$ and $\lambda_{n}=i \omega_{n}=i\left(\omega_{n r}+i \omega_{n i}\right)$ are respectively the complex amplitude, $n$th eigenmode and $n$th eigenvalue and C.C. denotes the complex conjugate of the proceeding term. $\omega_{n r}$ is the natural frequency and $\omega_{n i}$ corresponds to the damping ratio. By substituting Eq. (6) into Eq. (4), we obtain the equation governing $\Phi_{n}(n=1,2, \cdots)$ as

$$
\begin{aligned}
\Phi_{n}^{\prime \prime \prime \prime}+\left\{U^{2}-\gamma\right. & (1-s)\} \Phi_{n}^{\prime \prime} \\
& +\left(2 i \omega_{n} \sqrt{\beta} U+\gamma\right) \Phi_{n}^{\prime}-\omega_{n}^{2} \Phi_{n}=0,
\end{aligned}
$$

where $\Phi_{n}$ can be complex. We can then express $\Phi_{n}(s)$. From the boundary conditions given as Eq. (5), we have the associated boundary conditions

$\left\{\begin{array}{l}\Phi_{n}(0)=0, \Phi_{n}^{\prime}(0)=0 \\ \Phi_{n}^{\prime \prime}(1)=0, \Phi_{n}^{\prime \prime \prime}(1)=0 .\end{array}\right.$

We express $\Phi_{n}$ using a power series $[26,29]$ as

$\Phi_{n}= \begin{cases}{ }^{0} \Phi_{n}=\sum_{j=0}^{\infty} \frac{a_{j}}{j !} s^{j} & (0 \leq s \leq 0.5) \\ { }^{1} \Phi_{n}=\sum_{j=0}^{\infty} \frac{b_{j}}{j !}(1-s)^{j} & (0.5 \leq s \leq 1) .\end{cases}$

From the boundary conditions, we have

$a_{0}=0, a_{1}=0$, 
$b_{2}=0, b_{3}=0$.

We then determine the coefficients $a_{j}$ and $b_{j}(j=$ $0,1,2, \cdots)$. We initially consider the mode in the range of $0 \leq s \leq 0.5$. Substituting Eq. (9) into Eq. (7), we obtain

$$
\begin{aligned}
& {\left[a_{4} s^{0}+a_{5} s^{1}+\cdots+\frac{1}{j !} a_{j+4} s^{j}+\cdots\right]} \\
& +\left(U^{2}-\gamma\right)\left[a_{2} s^{0}+a_{3} s^{1}+\cdots+\frac{1}{j !} a_{j+2} s^{j}+\cdots\right] \\
& +\gamma\left[a_{2} s^{1}+a_{3} s^{2}+\cdots+\frac{1}{(j-1) !} a_{j+1} s^{j}+\cdots\right] \\
& +\left(2 i \omega_{n} \sqrt{\beta} U+\gamma\right) \\
& {\left[a_{1} s^{0}+a_{2} s^{1}+\cdots+\frac{1}{j !} a_{j+1} s^{j}+\cdots\right]} \\
& -\omega_{n}^{2}\left(a_{0} s^{0}+a_{1} s^{1}+\cdots+\frac{a_{j}}{j !} s^{j}+\cdots\right)=0 .
\end{aligned}
$$

Equating the coefficients of like powers of $s$ yields

$$
\begin{aligned}
s^{0}: & a_{4}+\left(U^{2}-\gamma\right) a_{2} \\
& +\left(2 i \omega_{n} \sqrt{\beta} U+\gamma\right) a_{1}-\omega_{n}^{2} a_{0}=0 \\
s^{1}: & a_{5}+\left(U^{2}-\gamma\right) a_{3} \\
& +\gamma a_{2}+\left(2 i \omega_{n} \sqrt{\beta} U+\gamma\right) a_{2}-\omega_{n}^{2} a_{1}=0
\end{aligned}
$$$$
s^{j}: \frac{1}{j !} a_{j+4}+\frac{\left(U^{2}-\gamma\right)}{j !} a_{j+2}+\frac{\gamma}{(j-1) !} a_{j+1}
$$$$
+\frac{2 i \omega_{n} \sqrt{\beta} U+\gamma}{j !} a_{j+1}-\frac{\omega_{n}^{2}}{j !} a_{j}=0 .
$$

Considering the boundary conditions given as Eq. (10), we express $a_{j}$ in Eq. (13) using only $a_{2}$ and $a_{3}$; we take the coefficients of $a_{2}$ and $a_{3}$ in $a_{j}$ as ${ }_{j}^{00} \Phi_{n}$ and ${ }_{j}^{01} \Phi_{n}$ respectively. We then obtain ${ }_{j}^{00} \Phi_{n}$ and ${ }_{j}^{01} \Phi_{n}$ from Eq. (13) as follows. For $0 \leq j \leq 3$ :

$\left\{\begin{array}{c}{ }_{0}^{00} \Phi_{n}=0,{ }_{0}^{01} \Phi_{n}=0 \\ { }_{1}^{00} \Phi_{n}=0,{ }_{1}^{01} \Phi_{n}=0 \\ { }_{2}^{00} \Phi_{n}=1,{ }_{2}^{01} \Phi_{n}=0 \\ { }_{3}^{00} \Phi_{n}=0,{ }_{3}^{01} \Phi_{n}=1 .\end{array}\right.$

For $j \geq 4$ :

$$
\begin{aligned}
{ }_{j}^{00} \Phi_{n} & =\left(\gamma-U^{2}\right){ }_{j-2}^{00} \Phi_{n} \\
& -\left\{2 i \omega_{n} \sqrt{\beta} U+\gamma(j-3)\right\}{ }_{j-3}^{00} \Phi_{n}+\omega_{n}^{2}{ }_{j-4}^{00} \Phi_{n}, \\
{ }_{j}^{01} \Phi_{n} & =\left(\gamma-U^{2}\right){ }_{j-2}^{01} \Phi_{n} \\
& -\left\{2 i \omega_{n} \sqrt{\beta} U+\gamma(j-3)\right\}{ }_{j-3}^{01} \Phi_{n}+\omega_{n}^{2}{ }_{j-4}^{01} \Phi_{n} .
\end{aligned}
$$

Using ${ }_{j}^{00} \Phi_{n}$ and ${ }_{j}^{01} \Phi_{n}$, we can rewrite ${ }^{0} \Phi_{n}$ in Eq. (9) as

$$
\begin{aligned}
{ }^{0} \Phi_{n}(s) & =a_{0} s^{0}+a_{1} s^{1}+\cdots+\frac{1}{j !} a_{j} s^{j}+\cdots \\
& =a_{2} \sum_{j=0}^{\infty} \frac{1}{j !}{ }_{j}^{00} \Phi_{n} s^{j}+a_{3} \sum_{j=0}^{\infty} \frac{1}{j !}{ }_{j}^{01} \Phi_{n} s^{j},
\end{aligned}
$$

and taking $\sum_{j=0}^{\infty} \frac{1}{j !}{ }_{j}^{00} \Phi_{n} s^{j}$ and $\sum_{j=0}^{\infty} \frac{1}{j !}{ }_{j}^{01} \Phi_{n} s^{j}$ as ${ }^{00} \Phi_{n}(s)$ and ${ }^{01} \Phi_{n}(s)$, we can express the $n$th eigenmode $\Phi_{n}(s)$ at $0 \leq s \leq 0.5$ as ${ }^{0} \Phi_{n}$ with the simple form

${ }^{0} \Phi_{n}(s)=a_{2}{ }^{00} \Phi_{n}(s)+a_{3}{ }^{01} \Phi_{n}(s)$.

We next consider the mode in the range of $0.5 \leq$ $s \leq 1$. We can determine the coefficients of the term $(1-s)^{j}$ of the $n$th eigenmode $\Phi_{n}$ at $0.5 \leq s \leq 1$ as $b_{j}$ in the same manner as for $a_{j}$. In this case, by substituting Eq. (9) into Eq. (7), we obtain

$$
\begin{aligned}
& (1-s)^{0}: b_{4}+U^{2} b_{2} \\
& -\left(2 i \omega_{n} \sqrt{\beta} U+\gamma\right) b_{1}-\omega_{n}^{2} b_{0}=0 \\
& (1-s)^{1}: b_{5}+U^{2} b_{3} \\
& -\gamma b_{2}-\left(2 i \omega_{n} \sqrt{\beta} U+\gamma\right) b_{2}-\omega_{n}^{2} b_{1}=0 \\
& (1-s)^{j}: \frac{1}{j !} b_{j+4}+\frac{U^{2}}{j !} b_{j+2}-\frac{\gamma}{(j-1) !} b_{j+1} \\
& -\frac{2 i \omega_{n} \sqrt{\beta} U+\gamma}{j !} b_{j+1}-\frac{\omega_{n}^{2}}{j !} b_{j}=0 .
\end{aligned}
$$

and taking into account the boundary conditions of Eq. (11), we can express $b_{j}$ in Eq. (19) using only $b_{0}$ and $b_{1}$; we take the coefficients of $b_{0}$ and $b_{1}$ in $b_{j}$ as ${ }_{j}^{10} \Phi_{n}$ and ${ }_{j}^{11} \Phi_{n}$, respectively. We then obtain ${ }_{j}^{10} \Phi_{n}$ and ${ }_{j}^{11} \Phi_{n}$ as $0 \leq j \leq 3$ :

$$
\left\{\begin{array}{c}
{ }_{0}^{10} \Phi_{n}=1,{ }_{0}^{11} \Phi_{n}=0 \\
{ }_{1}^{10} \Phi_{n}=0,{ }_{1}^{11} \Phi_{n}=1 \\
{ }_{2}^{10} \Phi_{n}=0,{ }_{2}^{11} \Phi_{n}=0 \\
{ }_{3}^{10} \Phi_{n}=0,{ }_{3}^{11} \Phi_{n}=0
\end{array}\right.
$$

$j \geq 4$ :

$$
\begin{aligned}
{ }_{j}^{10} \Phi_{n} & =-U^{2}{ }_{j-2}^{10} \Phi_{n} \\
& +\left\{2 i \omega_{n} \sqrt{\beta} U+\gamma(j-3)\right\}_{j-3}^{10} \Phi_{n}+\omega_{i j-4}^{2} \Phi_{n}, \\
{ }_{j}^{11} \Phi_{n} & =-U^{2}{ }_{j-2}^{11} \Phi_{n} \\
& +\left\{2 i \omega_{n} \sqrt{\beta} U+\gamma(j-3)\right\}{ }_{j-3}^{11} \Phi_{n}+\omega_{n j-4}^{2} \Phi_{n} .
\end{aligned}
$$


Letting $\sum_{j=0}^{\infty} \frac{1}{j !}{ }_{j}^{10} \Phi_{n}(1-s)^{j}$ and $\sum_{j=0}^{\infty} \frac{1}{j !}{ }_{j}^{11} \Phi_{n}(1-s)^{j}$ be ${ }^{10} \Phi_{n}(s)$ and ${ }^{11} \Phi_{n}(s)$, respectively, we can express the $n$th eigenmode $\Phi_{n}$ at $0.5 \leq s \leq 1$ as ${ }^{1} \Phi_{n}(s)$ by

${ }^{1} \Phi_{n}(s)=b_{0}{ }^{10} \Phi_{n}(s)+b_{1}{ }^{11} \Phi_{n}(s)$.

We impose the four conditions that ${ }^{0} \Phi_{n},{ }^{1} \Phi_{n}$ and their derivatives from first to third order are the same at $s=0.5$ :

$\left\{\begin{array}{l}{ }^{0} \Phi_{n}(0.5)={ }^{1} \Phi_{n}(0.5) \\ { }^{0} \Phi_{n}^{\prime}(0.5)={ }^{1} \Phi_{n}^{\prime}(0.5) \\ { }^{0} \Phi_{n}^{\prime \prime}(0.5)={ }^{1} \Phi_{n}^{\prime \prime}(0.5) \\ { }^{0} \Phi_{n}^{\prime \prime \prime}(0.5)={ }^{1} \Phi_{n}^{\prime \prime \prime}(0.5) .\end{array}\right.$

These equations are expressed as homogeneous equations in terms of $a_{2}, a_{3}, b_{0}$ and $b_{1}$ :

$$
\left[\begin{array}{cccc}
{ }^{00} \Phi_{n}(0.5) & { }^{01} \Phi_{n}(0.5) & -{ }^{11} \Phi_{n}(0.5) & -{ }^{11} \Phi_{n}(0.5) \\
{ }^{00} \Phi_{n}^{\prime}(0.5) & { }^{01} \Phi_{n}^{\prime}(0.5) & -{ }^{11} \Phi_{n}^{\prime}(0.5) & -{ }^{11} \Phi_{n}^{\prime}(0.5) \\
{ }^{00} \Phi_{n}^{\prime \prime}(0.5) & { }^{01} \Phi_{n}^{\prime \prime}(0.5) & -{ }^{11} \Phi_{n}^{\prime \prime}(0.5) & -{ }^{11} \Phi_{n}^{\prime \prime}(0.5) \\
{ }^{00} \Phi_{n}^{\prime \prime \prime}(0.5) & { }^{01} \Phi_{n}^{\prime \prime \prime}(0.5)-{ }^{11} \Phi_{n}^{\prime \prime \prime}(0.5) & -{ }^{11} \Phi_{n}^{\prime \prime \prime}(0.5)
\end{array}\right]\left[\begin{array}{c}
a_{2} \\
a_{3} \\
b_{0} \\
b_{1}
\end{array}\right]
$$

To have nontrivial solutions for $a_{2}, a_{3}, b_{0}$ and $b_{1}$, the determinant of the coefficient matrix in Eq. (25) must be zero; because the imaginary and real parts must be zero, we have two real equations for the condition. By solving these two equations, we obtain $\omega_{n r}$ and $\omega_{n i}$ for the $n$th mode. We can then obtain the complex-valued coefficients of each power of $s$ in Eqs. (18) and (23) and describe the real and imaginary components of the complex eigenmode.

Considering the terms up to 80th order of the power series, we obtain from Eq. (25) $\omega_{n r}$ and $\omega_{n i}$ for the first, second and third modes. Figure 2 shows the changes in $\omega_{r}$ and $\omega_{i}$ depending on $U$ for the first, second and third modes, where $\beta=0.388$ and $\gamma=74.2$ corresponding to the experimental parameter values of Pipe 1 in section 5. The numbers in the figure are values of the non-dimensional flow velocity $U$. The first and second modes are stable for any fluid velocity because $\omega_{i}>0$ independent of $U$. Unlike the case for the first and second modes, $\omega_{i}$ of the third mode becomes zero from positive values at $U=11.8 \equiv U_{c r}$ when $U$ increases. The system is destabilized and undergoes self-excited oscillation with the third mode at $U>U_{c r}$.

At the critical point, we obtain ${ }^{00} \Phi,{ }^{01} \Phi,{ }^{10} \Phi$ and ${ }^{11} \Phi$ and a pair of $a_{2}, a_{3}, b_{0}$ and $b_{1}$ from Eq. (25). We obtain the theoretical third mode shape from these values. This mode is a complex function with respect to the arclength $s$, and the real and imaginary components of the mode shape are presented in Fig. 3(a) and (b),

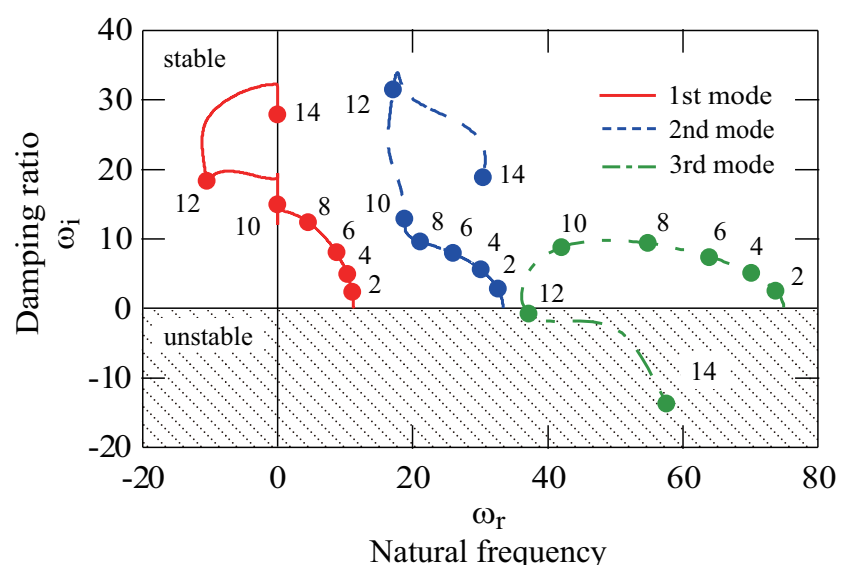

Fig. 2 Argand diagram for $\beta=0.388$ and $\gamma=74.2$. The vertical and horizontal axes respectively give the real and imaginary parts of $\omega$. The numbers in the figure are values of the non-dimensional fluid velocity $U . \omega_{i}$ of the third mode (dashed and dotted line) becomes zero from positive values at $U=11.8 \equiv U_{c r}$, in contrast with that of the first (solid line) and second (dashed line) modes. The system is destabilized and undergoes self-excited oscillation with the third mode in the range of $U>U_{c r}$.
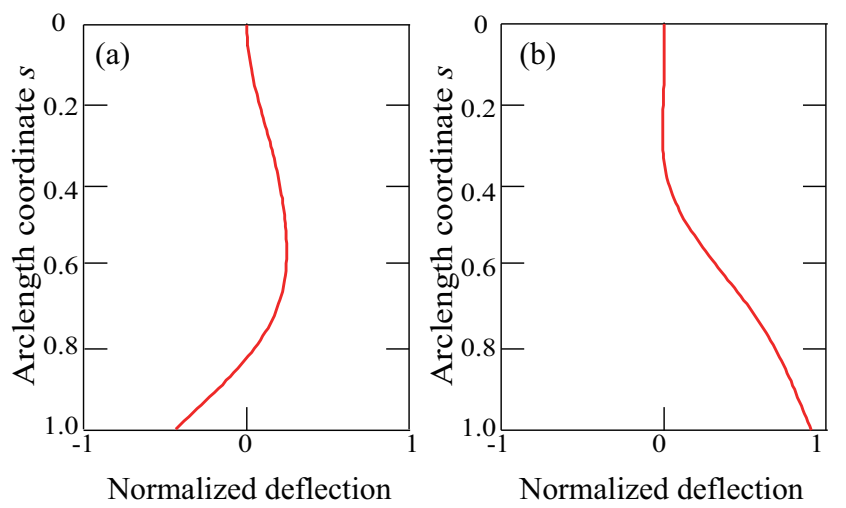

Fig. 3 Theoretical third complex mode shape of the pipe in the case that $\beta=0.388$ and $\gamma=74.2$ ((a) Real component of the mode and (b) imaginary component of the mode). Each mode shape is normalized by the absolute value $\sqrt{\Phi_{r}^{2}+\Phi_{i}^{2}}$ of the complex number $\Phi$ at the end point $(s=1)$.

respectively, where the horizontal and vertical axes respectively give the normalized deflection and archlength $s$. In the figure, the mode shapes are normalized by the absolute value $\sqrt{\Phi_{r}^{2}(1)+\Phi_{i}^{2}(1)}$ of $\Phi$ at $s=1$.

\section{Complex eigenmodes for nonlinear steady-state self-excited oscillation}

Cantilevered elastic pipes conveying fluid essentially undergo self-excited oscillation due to the non-selfadjointness. Additionally, because the non-selfadjointness can make the eigenmode complex, the proposition of a method of 
detecting complex eigenmodes (i.e., the real and imaginary components of the eigenmodes) from experimental data enables a deeper understanding of self-excited oscillation due to the non-selfadjointness of systems from an experimental point of view.

Incidentally, it is theoretically predicted that the amplitude of the self-excited oscillation transiently grows with time. In practical systems, because nonlinear components of the restoring and inertia forces of a pipe, which are expressed by Eq. (2), necessarily exist, there can be a limit cycle (i.e., a self-excited oscillation with a constant amplitude) after the transient state. In adopting the experimental identification method of complex modes proposed in section 4 , it is preferable to use the time history data for the steady state because the time history data for the transient state can include modes other than the targeted and destabilized mode.

This section examines the contribution of the cubic nonlinear components, which are responsible for the steady state in the self-excited oscillation, to the eigenmode. It is clarified that the mode in the steady-state self-excited oscillation resulting from the nonlinearities mentioned above can deviate from $\Phi_{n}$ in section 2.2, which is obtained under the assumption of linearization.

To examine the steady-state self-excited oscillation with a constant amplitude, we consider the nonlinear effects expressed in Eq. (2), which are neglected in section 2. To focus on the behavior in the neighborhood of the critical velocity for the $n$th mode, $U=U_{n c r}$, we introduce the detuning parameter $\Delta U$, which is assumed small, as

$U=U_{n c r}+\Delta U_{n}=U_{n c r}+\epsilon \Delta \hat{U}_{n}$

where $\epsilon$ is a small parameter and $\Delta \hat{U}_{n}=O(1)$. Additionally, we assume the solution to have a uniform expansion:

$v=\epsilon^{\frac{1}{2}} \hat{v}_{n 1}+\epsilon^{\frac{3}{2}} \hat{v}_{n 3}+\cdots$.

Furthermore, we introduce the multiple time scales $t_{0}=$ $t$ and $t_{1}=\epsilon t$. Substituting Eqs. (26) and (27) into Eqs. (2) and (3) and equating the coefficients of like powers of $\epsilon$ yields

$$
\begin{aligned}
\epsilon^{\frac{1}{2}}: & \hat{v}_{n 1}^{\prime \prime \prime \prime}+\left\{U_{n c r}^{2}-\gamma(1-s)\right\} \hat{v}_{n 1}^{\prime \prime} \\
& 2 \sqrt{\beta} U_{n c r} D_{0} \hat{v}_{n 1}^{\prime}+\gamma \hat{v}_{n 1}^{\prime}+D_{0}^{2} \hat{v}_{n 1}=0
\end{aligned}
$$

$s=0: v_{n 1}(0)=0, v_{n 1}^{\prime}(0)=0$,

$s=1: v_{n 1}^{\prime \prime}(1)=0, v_{n 1}^{\prime \prime \prime}(1)=0$,

$$
\begin{aligned}
\epsilon^{\frac{3}{2}}: & \hat{v}_{n 3}^{\prime \prime \prime \prime}+\left\{U_{n c r}^{2}-\gamma(1-s)\right\} \hat{v}_{n 3}^{\prime \prime} \\
& +2 \sqrt{\beta} U_{n c r} D_{0} \hat{v}_{n 3}^{\prime}+\gamma \hat{v}_{n 3}^{\prime}+D_{0}^{2} \hat{v}_{n 3} \\
& =-2 \sqrt{\beta} U_{n c r} D_{1} \hat{v}_{n 1}^{\prime}-2 \sqrt{\beta} \Delta \hat{U}_{n} D_{0} \hat{v}_{n 1}^{\prime} \\
& -2 D_{0} D_{1} \hat{v}_{n 1}-2 \Delta \hat{U}_{n} U_{n c r} \hat{v}_{n 1}^{\prime \prime}+n\left(\hat{v}_{n 1}^{3}\right),
\end{aligned}
$$

$s=0: v_{n 3}(0)=0, v_{n 3}^{\prime}(0)=0$

$s=1: v_{n 3}^{\prime \prime}(1)=0, v_{n 3}^{\prime \prime \prime}(1)=0$,

where $D_{0}=\frac{\partial}{\partial t_{0}}$ and $D_{1}=\frac{\partial}{\partial t_{1}}$. In this paper, we consider the self-excited oscillation not with multiple modes but with a single $n$th mode. We let $\hat{v}_{n 1}$ and $\hat{v}_{n 3}$ be

$\left\{\begin{array}{l}\hat{v}_{n 1}=A_{n}\left(t_{1}\right) \Phi_{n 1}(s) \mathrm{e}^{\lambda_{n} t_{0}}+C . C . \\ \hat{v}_{n 3}=\Phi_{n 3}\left(s, t_{1}\right) \mathrm{e}^{\lambda_{n} t_{0}}+C . C .\end{array}\right.$

and by substituting these into Eqs. (28) to (31), we obtain equations that are satisfied by $\Phi_{n 1}$ and $\Phi_{n 3}$ :

$$
\begin{aligned}
\epsilon^{\frac{1}{2}}: \Phi_{n 1}^{\prime \prime \prime \prime} & +\left\{U_{n c r}^{2}-\gamma(1-s)\right\} \Phi_{n 1}^{\prime \prime} \\
& +\left(2 i \omega_{n} \sqrt{\beta} U_{n c r}+\gamma\right) \Phi_{n 1}^{\prime}-\omega_{n}^{2} \Phi_{n 1}=0, \\
\Phi_{n 1}(0)= & 0, \Phi_{n 1}^{\prime}(0)=0 \\
\Phi_{n 1}^{\prime \prime}(1)= & 0, \Phi_{n 1}^{\prime \prime \prime}(1)=0, \\
\epsilon^{\frac{3}{2}}: \Phi_{n 3}^{\prime \prime \prime \prime} & +\left\{U_{n c r}^{2}-\gamma(1-s)\right\} \Phi_{n 3}^{\prime \prime} \\
& +\left(2 i \omega_{n} \sqrt{\beta} U_{n c r}+\gamma\right) \Phi_{n 3}^{\prime}-\omega_{n}^{2} \Phi_{n 3} \\
= & -2 \sqrt{\beta} U_{n c r} D_{1} A_{n} \Phi_{n 1}^{\prime}-2 i \omega_{n} D_{1} A_{n} \Phi_{n 1} \\
\quad- & 2 \Delta \hat{U}_{n} U_{n c r} A_{n} \Phi_{n 1}^{\prime \prime}-2 i \omega_{n} \sqrt{\beta} \Delta \hat{U}_{n} A_{n} \Phi_{n 1}^{\prime} \\
& +\left|A_{n}\right|^{2} A_{n} n\left(\Phi_{n 1}\right), \\
\Phi_{n 3}(0) & =0, \Phi_{n 3}^{\prime}(0)=0 \\
\Phi_{n 3}^{\prime \prime}(1) & =0, \Phi_{n 3}^{\prime \prime \prime}(1)=0
\end{aligned}
$$

where $n\left(\Phi_{n 1}\right)$ is given in Appendix A. Because Eq. (33) is equivalent to Eq. (7), $\Phi_{n 1}$ corresponds to $\Phi_{n}$ in section 2. Equations (33) and (34) are non-selfadjoint, and thus, to obtain the solvability condition [16] of $\Phi_{n 3}$, we need to use the adjoint function $\Psi_{n 1}$ with respect to $\Phi_{n}$, which is discussed in detail in Appendix B. Multiplying Eq. (35) by the adjoint function $\Psi_{1}$ (see Appendix B) and integrating the result over the interval from 0 to 1 with respect to $s$ yields

$$
\begin{aligned}
& \int_{0}^{1}\left[\Phi_{n 3}^{\prime \prime \prime \prime}+\left\{U_{n c r}^{2}-\gamma(1-s)\right\} \Phi_{n 3}^{\prime \prime}\right. \\
& \left.+\left(2 i \omega_{n} \sqrt{\beta} U_{n c r}+\gamma\right) \Phi_{n 3}^{\prime}-\omega_{n}^{2} \Phi_{n 3}\right] \bar{\Psi}_{n 1} d s \\
& =\int_{0}^{1}\left[-2 \sqrt{\beta} U_{n c r} D_{1} A_{n} \Phi_{n 1}^{\prime}-2 i \omega_{n} D_{1} A_{n} \Phi_{n 1}\right. \\
& -2 \Delta \hat{U}_{n} U_{n c r} A_{n} \Phi_{n 1}^{\prime \prime}-2 i \omega_{n} \sqrt{\beta} \Delta \hat{U}_{n} A_{n} \Phi_{n 1}^{\prime} \\
& \left.+\left|A_{n}\right|^{2} A_{n} n\left(\Phi_{n 1}\right)\right] \bar{\Psi}_{n 1} d s .
\end{aligned}
$$


Considering the boundary conditions given in Eq. (B.3), Eq. (37) leads to

$D_{1} A_{n}=\frac{K_{1}}{K_{0}} \Delta \hat{U}_{n} A_{n}+\frac{K_{2}}{K_{0}}\left|A_{n}\right|^{2} A_{n}$,

where

$K_{0}=\int_{0}^{1}\left(-2 \sqrt{\beta} U_{n c r} \Phi_{n 1}^{\prime}-2 i \omega_{n} \Phi_{n 1}\right) \bar{\Psi}_{n 1} d s$

$K_{1}=\int_{0}^{1}\left(2 U_{n c r} \Phi_{n 1}^{\prime \prime}+2 i \omega_{n} \sqrt{\beta} \Phi_{n 1}^{\prime}\right) \bar{\Psi}_{n 1} d s$

$K_{2}=-\int_{0}^{1} n\left(\Phi_{n 1}\right) \bar{\Psi}_{n 1} d s$

Substituting $A_{n}=\frac{\hat{a}}{2} \mathrm{e}^{i \phi}$ into Eq. (38), we obtain equations expressing the time variation of the absolute value and the argument of the complex amplitude $A_{n}$ as

$\frac{d a}{d t}=k_{1 r} \Delta U_{n} a+\frac{1}{4} k_{2 r} a^{3}$,

$\frac{d \phi}{d t}=k_{1 i} \Delta U_{n}+\frac{1}{4} k_{2 i} a^{2}$,

where $a=\epsilon^{\frac{1}{2}} \hat{a}$ and

$k_{1 r}=\operatorname{Re}\left(\frac{\mathrm{K}_{1}}{\mathrm{~K}_{0}}\right), \mathrm{k}_{1 \mathrm{i}}=\operatorname{Im}\left(\frac{\mathrm{K}_{1}}{\mathrm{~K}_{0}}\right)$

$k_{2 r}=\operatorname{Re}\left(\frac{\mathrm{K}_{2}}{\mathrm{~K}_{0}}\right), \mathrm{k}_{2 \mathrm{i}}=\operatorname{Im}\left(\frac{\mathrm{K}_{2}}{\mathrm{~K}_{0}}\right)$.

Letting $\frac{d a}{d t}=0$ in Eq. (40) gives the steady-state amplitude as

$a_{s t}=0, \sqrt{\frac{-4 k_{1 r} \Delta U_{n}}{k_{2 r}}}$.

It is known that supercritical Hopf bifurcation occurs if a pipe is sufficiently slender [3]. Thus, in this study, because we consider the pipe as slender, $k_{1 r} k_{2 r}<0$ and we obtain the bifurcation diagram with the control parameter $\Delta U$ shown in Fig. 5 using Eq. (43). The trivial steady-state amplitude $a_{s t}=0$ is stable when $\Delta U_{n}$ is negative. When $\Delta U_{n}$ becomes positive, the trivial steady-state amplitude becomes unstable and the amplitude increases until the magnitude reaches the nontrivial steady-state amplitude $a_{s t}=\sqrt{\frac{-4 k_{1 r} \Delta U_{n}}{k_{2 r}}}$.

In the steady state, $v$ is obtained from Eqs. (27) and (32) as

$v=\frac{a_{s t}}{2} \Phi_{n 1}(s) \mathrm{e}^{i\left(\omega_{r} t+\phi\right)}+C . C .+O\left(\epsilon^{\frac{3}{2}}\right)$,

where $\Phi_{n 1}(s)$ can be complex and expressed as $\Phi_{n 1}(s)=$ $\Phi_{n r}(s)+i \Phi_{n i}(s)$. In the absence of a nonconservative force, $\Phi_{n}(s)$ is real and $\Phi_{n i}(s)=0$ at all $s$. The leadingorder term is expressed by the linear mode derived in section 2. Therefore, the contribution of the nonlinearity of the system to the mode is very small and $O\left(\epsilon^{\frac{3}{2}}\right)$. We can regard the mode of self-excited vibration in the steady state, which is experimentally observed in the steady state, as the linear mode approximately.

Substituting $\Phi_{n}(s)=\Phi_{n r}(s)+i \Phi_{n i}(s)$ into Eq. (44), we obtain

$$
\begin{aligned}
v=a_{s t}\left\{\Phi_{n r}(s) \cos \left(\omega_{r} t+\phi\right)\right. \\
\left.-\Phi_{n i}(s) \sin \left(\omega_{r} t+\phi\right)\right\}+O\left(\epsilon^{\frac{3}{2}}\right) \\
=a_{s t}\left|\Phi_{n}(s)\right| \cos \left\{\omega_{r} t+\phi+\psi(s)\right\}+O\left(\epsilon^{\frac{3}{2}}\right) \\
=a_{s t}\left\{\left|\Phi_{n}(s)\right| \cos \psi(s) \cos \left(\omega_{r} t+\phi\right)\right. \\
\left.-\left|\Phi_{n}(s)\right| \sin \psi(s) \sin \left(\omega_{r} t+\phi\right)\right\},
\end{aligned}
$$

where

$$
\begin{aligned}
& \cos \psi(s)=\frac{\Phi_{n r}(s)}{\sqrt{\Phi_{n r}^{2}(s)+\Phi_{n i}^{2}(s)}}, \\
& \sin \psi(s)=\frac{\Phi_{n i}(s)}{\sqrt{\Phi_{n r}^{2}(s)+\Phi_{n i}^{2}(s)}}, \\
& \left|\Phi_{n}(s)\right|=\sqrt{\Phi_{n r}^{2}(s)+\Phi_{n i}^{2}(s)} .
\end{aligned}
$$

From Eq. (45), we can express $\Phi_{n r}(s)$ and $\Phi_{n i}(s)$ as

$\Phi_{n r}(s)=\left|\Phi_{n}(s)\right| \cos \psi(s)$,

$\Phi_{n i}(s)=\left|\Phi_{n}(s)\right| \sin \psi(s)$.

Therefore, because the mode is complex, as seen from Eq. (45), the phase $\omega_{r} t+\phi+\psi(s)$ depends on not only time $t$ but also arclength $s$, that is, the position of the pipe. We cannot determine the eigenmode from the deflection shape of the pipe at a moment in time in stroboscopic photographs of the vibration . Figure 4 presents stroboscopic photographs of Pipe 1 obtained in the experiment described in section 5 ; the vertical line corresponds to the $x$ axis in theory and is the position of the pipe in its rest state. The photographs were taken at $1 / 6$ intervals of the period. A blue mark was placed on the upper pipe and a red mark on the lower pipe for the observation of motion. The figure shows that both move in the same direction in Figs. 4 (a)-(c) and (d)-(f). Meanwhile, in Fig. 4 (c) and (d), we see that the absolute value of the displacement of the upper mark decreases while that of the lower mark increases. Such motion cannot be explained by any real modes and indicates that the eigenmode is complex and the phase depends on the arclength $s$ as mentioned above; the characteristic motion corresponds to that reported by Gregory and Paidoussis [9]. It is impossible to identify the real and imaginary components directly from such stroboscopic photographs. We therefore propose a method of detecting the real and imaginary components of the complex eigenmode from experimentally 


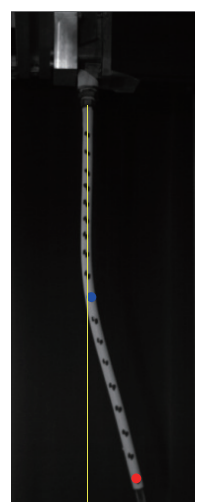

(a)

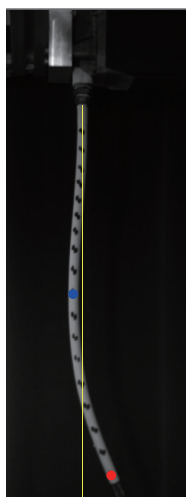

(b)

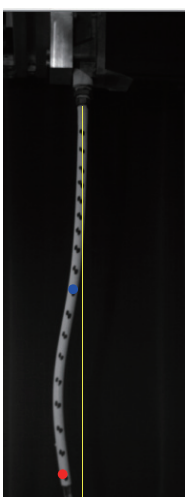

(c)

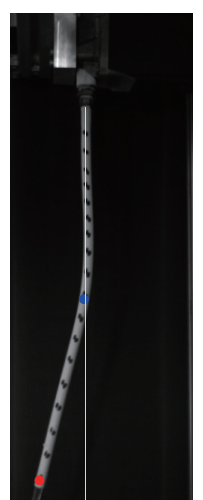

(d)

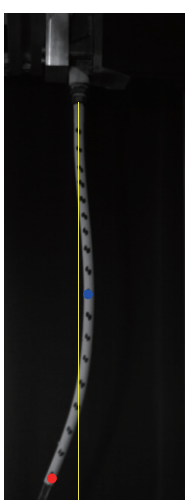

(e)

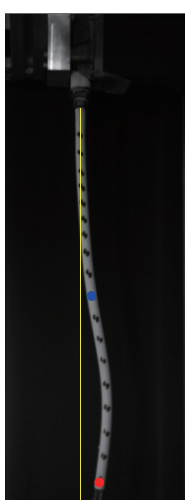

(f)

Fig. 4 Stroboscopic photographs of the vibration of Pipe 1. These photographs were taken at 1/6 intervals of the period . The vertical line indicates the $x$ axis and the position of the pipe in its rest state. In (a)-(c) and (d) $-(\mathrm{f})$, the two marks placed on the pipe move in the same direction. In (c)-(d), the absolute value of the displacement of the upper mark decreases while that of the lower dot increases, which is a characteristic of the complex mode and different from the motion of the real mode.

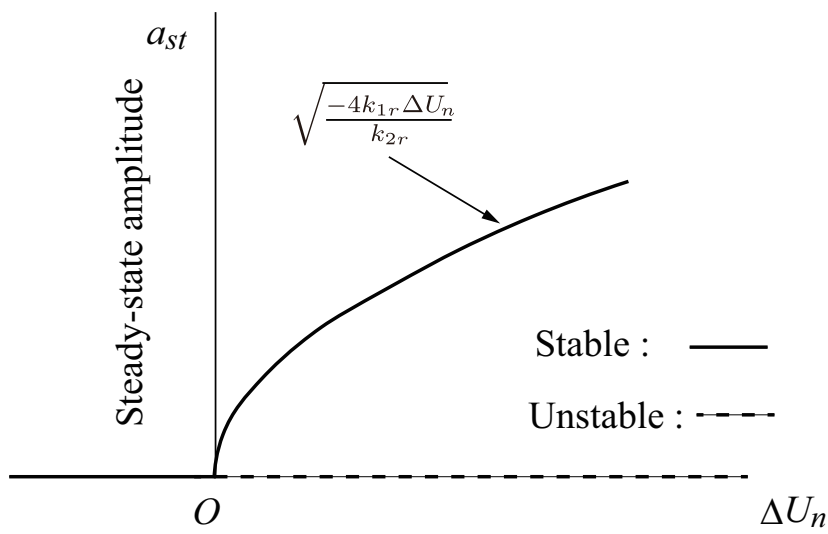

Deviation from critical flow velocity

Fig. 5 Bifurcation diagram. Solid and dashed lines respectively express the stable and unstable steady-state amplitudes. When $\Delta U_{n}<0$, the trivial steady-state amplitude $a_{s t}=0$ is stable. When $\Delta U_{n}>0$, the trivial steadystate amplitude becomes unstable and the stable nontrivial steady-state amplitude emerges. The amplitude reaches the nontrivial steady-state amplitude after the transient state $a_{s t}=\sqrt{\frac{-4 k_{1 r} \Delta U_{n}}{k_{2 r}}}$.

obtained time histories of the steady-state self-excited oscillation in the next section.

\section{Method of experimentally identifying the complex eigenmode}

As shown in section 3 , the nonlinear effects play a role in determining the magnitude of a steady-state amplitude but hardly modify the linear mode shape. Therefore, using the experimentally obtained steady-state self-excited oscillations, we propose a method of experimentally identifying the real and imaginary components of the linear complex mode, that is, $\Phi_{n r}(s)$ and $\Phi_{n i}(s)$ in Eq. (45).

Eq. (2) is autonomous, and thus without loss of generality, we can introduce a new time $t_{e}=t+\frac{\phi+\psi\left(s_{0}\right)}{\omega}$ so that the initial phase $\psi(s)+\phi$ at a point $s=s_{0}$ is zero. Substituting $t=t_{e}-\frac{\phi+\psi\left(s_{0}\right)}{\omega}$ into Eq. (45), we obtain

$$
\begin{aligned}
v= & a\left|\Phi_{n}(s)\right|\{\cos \psi(s) \cos (\omega t+\phi) \\
& \quad-\sin \psi(s) \sin (\omega t+\phi)\} \\
= & a\left|\Phi_{n}(s)\right|\left\{\cos \Delta \psi(s) \cos \omega t_{e}-\sin \Delta \psi(s) \sin \omega t_{e}\right\} \\
= & a\left|\Phi_{n}(s)\right| \cos \left\{\omega t_{e}+\Delta \psi(s)\right\} \\
= & a\left\{\Phi_{n r}^{\prime}(s) \cos \left(\omega t_{e}\right)-\Phi_{n i}^{\prime} \sin \left(\omega t_{e}\right)\right\}
\end{aligned}
$$

where $\Delta \psi(s)=\psi(s)-\psi\left(s_{0}\right), \Phi_{n r}^{\prime}(s)=\left|\Phi_{n}(s)\right| \cos \Delta \psi(s)$ and $\Phi_{n i}^{\prime}(s)=\left|\Phi_{n}(s)\right| \sin \Delta \psi(s)$. Hereafter, we omit the $(')$ notation for simplicity. By placing $\mathrm{N}$ measurement markers on the pipe, we obtain $\mathrm{N}$ time histories in the experiment as shown in section 5 . The displacements at the $n$th $(1 \leq n \leq N)$ marker $v_{e n}$ are expressed as

$$
\begin{gathered}
v_{e 1}\left(s_{1}, t_{e}\right)=a\left|\Phi_{e n}\left(s_{1}\right)\right| \cos \left(\omega t_{e}+\Delta \psi_{1 k}\right), \\
v_{e 2}\left(s_{2}, t_{e}\right)=a\left|\Phi_{e n}\left(s_{2}\right)\right| \cos \left(\omega t_{e}+\Delta \psi_{2 k}\right), \\
\vdots \\
v_{e N}\left(s_{N}, t_{e}\right)=a\left|\Phi_{e n}\left(s_{N}\right)\right| \cos \left(\omega t_{e}+\Delta \psi_{N k}\right),
\end{gathered}
$$

where $\Delta \psi_{i k}=\psi_{e}\left(s_{i}\right)-\psi_{e}\left(s_{k}\right)$ and $\Phi_{e n}, \psi_{e}$ are the $n$th eigenmode and phase obtained from the experiment. We select $s=s_{1}$ as the reference point of the phase, that is, $\Delta \psi_{1 k}=0$. Then, letting $\boldsymbol{v}_{\boldsymbol{e}}=\left(v_{e 1} v_{e 2} \cdots v_{e n}\right)^{t}$, 
we obtain

$$
\begin{array}{r}
\boldsymbol{v}_{\boldsymbol{e}}=a\left(\begin{array}{c}
\left|\Phi_{e n}\left(s_{1}\right)\right| \\
\left|\Phi_{e n}\left(s_{2}\right)\right| \cos \Delta \psi_{21} \\
\vdots \\
\left|\Phi_{e n}\left(s_{N}\right)\right| \cos \Delta \psi_{N 1}
\end{array}\right) \cos \omega t_{e} \\
-a\left(\begin{array}{c}
\left|\Phi_{e n}\left(s_{2}\right)\right| \sin \Delta \psi_{21} \\
\vdots \\
\left|\Phi_{e n}\left(s_{N}\right)\right| \sin \Delta \psi_{N 1}
\end{array}\right) \sin \omega t_{e} .
\end{array}
$$

Comparing Eq. (50) with Eq. (48), we can regard the coefficients of $\cos \omega t_{e}$ and $\sin \omega t_{e}$ in Eq. (50) as $\Phi_{n r}(s)$ and $\Phi_{n i}(s)$, respectively. We then express the real and imaginary components of the complex eigenmode obtained in the experiment, $\Phi_{e r}$ and $\Phi_{e i}$, as

$\Phi_{e r}=\left(\begin{array}{c}\left|\Phi_{e n}\left(s_{1}\right)\right| \\ \left|\Phi_{e n}\left(s_{2}\right)\right| \cos \Delta \psi_{21} \\ \vdots \\ \left|\Phi_{e n}\left(s_{N}\right)\right| \cos \Delta \psi_{N 1}\end{array}\right)$,

$\Phi_{e i}=\left(\begin{array}{c}0 \\ \left|\Phi_{e n}\left(s_{2}\right)\right| \sin \Delta \psi_{21} \\ \vdots \\ \left|\Phi_{e n}\left(s_{N}\right)\right| \sin \Delta \psi_{N 1}\end{array}\right)$.

\section{Experimental identification of the complex mode shape}

\subsection{Experimental setup}

We show the experimental apparatus in Figs. 6 and 7. We used three pipes whose parameters are given in Table 1 . Pipes 2 and 3 were the same pipe but Pipe 3 was attached to two brass rods with a diameter of $0.8 \mathrm{~mm}$ to increase its flexural rigidity. Pipes 1 and 2 underwent self-excited vibration in the third mode but had different critical speeds whereas Pipe 3 underwent self-excited vibration in the second mode. Flexural rigidity was identified experimentally using the method described in Appendix B. To obtain the mode shape experimentally, we placed 18 or 19 markers on the pipe as shown in Fig. 8 and recorded the time history of the steady-state self-excited oscillation using a high-speed camera (Photron: FASTCAM APX RS) after sufficient time had passed because in the steady state, the oscillation components relating to modes other than the focused self-excited mode decay. The number of frames per second, averaged marker interval, spatial resolution and magnitude of the normalized resolution are given in Table 2. From the recorded video, we derive
Table 1 Parameters of the three pipes used in the experiment

\begin{tabular}{clll}
\hline Parameter & Pipe 1 & Pipe 2 & Pipe 3 \\
\hline Length $[\mathrm{m}]$ & 0.393 & 0.396 & 0.396 \\
$\beta$ & 0.388 & 0.289 & 0.263 \\
$\gamma$ & 74.2 & 53.3 & 14.6 \\
\hline
\end{tabular}

Table 2 Shooting conditions for the three pipes

\begin{tabular}{clll}
\hline \multicolumn{1}{c}{ Parameter } & Pipe 1 & Pipe 2 & Pipe 3 \\
\hline Number of frames $[\mathrm{fps}]$ & 1500 & 1500 & 1500 \\
Average marker interval $[\mathrm{mm}]$ & 2.16 & 2.18 & 2.05 \\
Spatial resolution $[\mathrm{mm}]$ & 0.800 & 0.581 & 0.699 \\
Normalized resolution $\left[\times 10^{3}\right]$ & 7.69 & 5.13 & 9.01 \\
\hline
\end{tabular}

the time history data of the motion at each marker and obtain $a\left|\Phi_{e n}\left(s_{n}\right)\right|$ and $\phi+\psi_{n}$ from section 4 by analyzing the time histories using a fast Fourier transformation. $a\left|\Phi_{e n}\left(s_{n}\right)\right|$ expresses the amplitude at each point in Eq. (48), and the phase difference between markers is obtained as $\phi+\psi_{n}$. We can therefore decompose the complex mode into real and imaginary components as in Eq. (51) experimentally.

In practice, the markers on the pipe have displacement in not only the $y$ direction but also the $x$ direction. We let $u$ be the displacement at $s$ in the $x$ direction. We express $u$ as

$$
\begin{aligned}
u & =\int_{0}^{s}\left(\sqrt{1-\left(\frac{\partial v}{\partial s}\right)^{2}}-1\right) d s \\
& \approx \frac{1}{2} \int_{0}^{s}\left(\frac{\partial v}{\partial s}\right)^{2} d s .
\end{aligned}
$$

From Eq. (52), we have $u=O\left(v^{2}\right)$. The displacement in the $x$ direction is much smaller than that in the $y$ direction and is considered negligible. Therefore, the lateral displacement in the $y$ direction at the point on the pipe whose distance from the supporting point along the curvilinear coordinate is $s$ is almost the same as that at the point on the pipe whose $x$ coordinate in Fig. 1 is $s$.

\subsection{Preliminary experiment}

In a preliminary investigation, we compared the theoretically and experimentally obtained critical flow speed and response frequency. These values are given in Table 3. Here, we compared the values for the self-excited oscillations of the second mode in Pipes 1 and 2 and the values for the self-excited oscillations of the third mode in Pipe 3. The differences between theoretical and experimental results are less than $6 \%$ for Pipes 1 and 2 and less than $10 \%$ for Pipe 3 . 
Table 3 Comparison of the frequencies of self-excited vibration and critical speed between experimental results and theoretical results

\begin{tabular}{lclllc}
\hline & Exp./Cal. & Pipe 1 & Pipe 2 & Pipe 3 & Unit \\
\hline Frequency & Exp. & 3.45 & 3.45 & 4.8 & $\mathrm{~Hz}$ \\
& Cal. & 3.38 & 3.67 & 5.26 & $\mathrm{~Hz}$ \\
Critical speed & Exp. & 4.24 & 4.79 & 8.25 & $\mathrm{~m} / \mathrm{s}$ \\
& Cal. & 4.31 & 5.02 & 8.74 & $\mathrm{~m} / \mathrm{s}$ \\
\hline
\end{tabular}

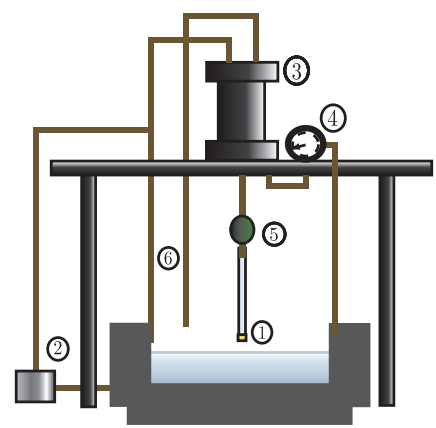

\begin{tabular}{|c|c|c|c|}
\hline 1 & Pipe & 4 & Pressure gauge \\
\hline 2 & Pump & 5 & Flowmeter \\
\hline 3 & Tank & 6 & Pressure control valve \\
\hline
\end{tabular}

Fig. 6 Experimental apparatus. Water stored in a lower tank is pumped up to a tank (3) by a pump (2). From the tank, the water is discharged through a pipe (1). We can change flow speed by turning a valve (6).

\subsection{Experimentally identified complex mode shapes}

In comparing the shapes of $\Phi_{e r}(s)$ and $\Phi_{e i}(s)$ obtained experimentally using the method proposed in section 4 with the shapes of $\Phi_{r}(s)$ and $\Phi_{i}(s)$ obtained theoretically from Eq. (45), we need to match the phase $\psi(s)$ of theoretical modes with that of experimental modes without loss of generality. Using the method described in section 2, we obtain the theoretical complex modes as a function of $s$. Thus, using Eq. (46), we obtain the phase $\psi(s)$ at all $s(0 \leq s \leq 1)$ and match the phase of the theoretical mode with that of the experimental mode. In the present study, we select the moving marker closest to the upper end of the pipe as the reference point.

First, the mode shapes of Pipe 1 obtained theoretically and experimentally are shown in Fig. 9, where (a) and (b) respectively show the real and imaginary components of the complex mode obtained experimentally using the method proposed in section 4 and (c) and (d)

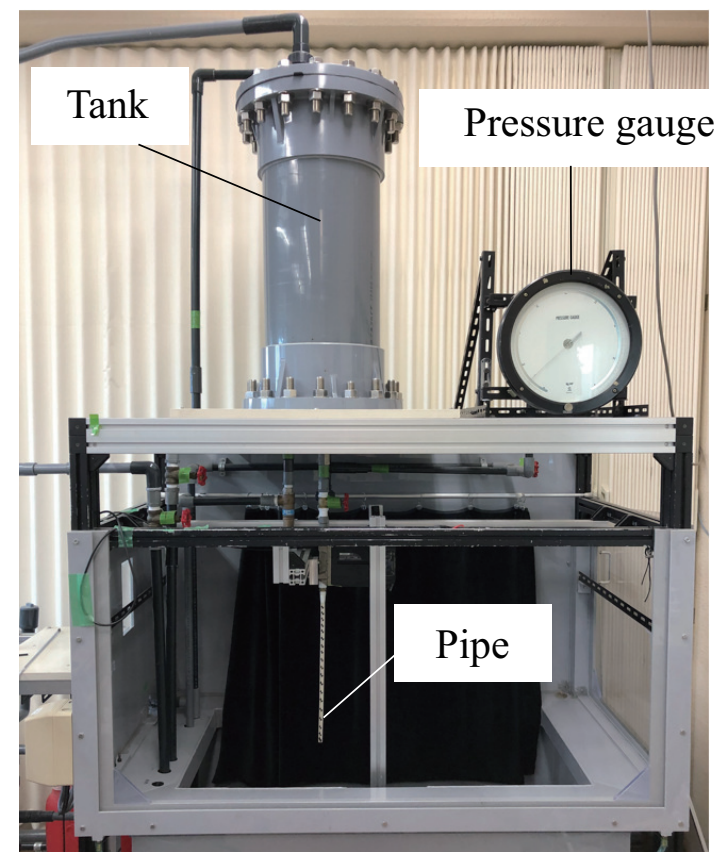

Fig. 7 Photograph of the experimental apparatus.

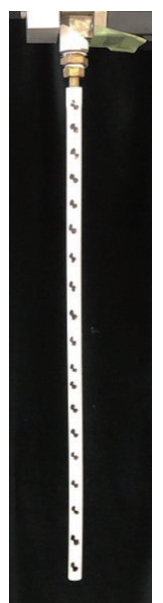

Fig. 8 Photograph of the pipe. Black markers on the pipe were used to obtain the mode shape. 

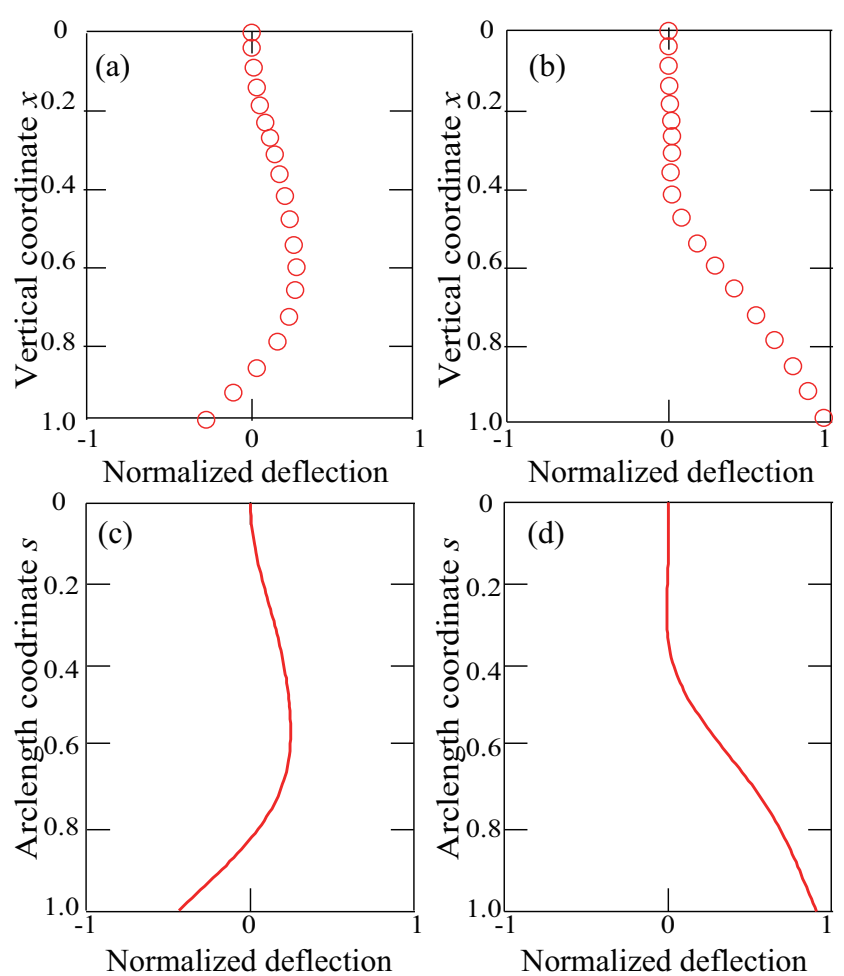

Fig. 9 Comparison of experimental and theoretical mode shapes for Pipe 1: (a) and (b) shapes of the experimental real and imaginary modes and (c) and (d) shapes of the theoretical real and imaginary modes.

show those obtained theoretically using the method presented in section 2. A comparison of (a) with (c) and a comparison of (b) with (d) shows that the shapes are in agreement qualitatively. That is to say, in a comparison of the real components (i.e., (a) and (c)), both shapes have a node and antinode on the lower pipe; the positions of the node and antinode are $x=0.869,0.598$ in (a) and $s=0.820,0.570$ in (c), respectively. The mode shapes are thus in good agreement qualitatively. In the comparison of the imaginary components, there is a node in the experimental mode shape (b) at $x=0.089$ whereas there are two nodes $s=0.090$ and 0.340 in the theoretical mode shape. On the basis of the results in section 4 , we introduce a new time $t_{e}$ such that the phases of the experimental and theoretical modes, $\psi_{e}+\phi$ and $\psi+\phi$, at the reference point are zero. The positions of the nodes of the experimental and theoretical modes should then be the same, but the positions of the experimental and theoretical nodes $x=0.089$ and $s=0.09$ deviate slightly. This slight deviation arises because we calculate the theoretical mode shape with a step size of 0.01 . That there is no node at $s=0.340$ in the experimental mode shape can be explained by a lack of measurement accuracy, that is, the gradient of the mode shape at $s=0.340$ is very small and, because
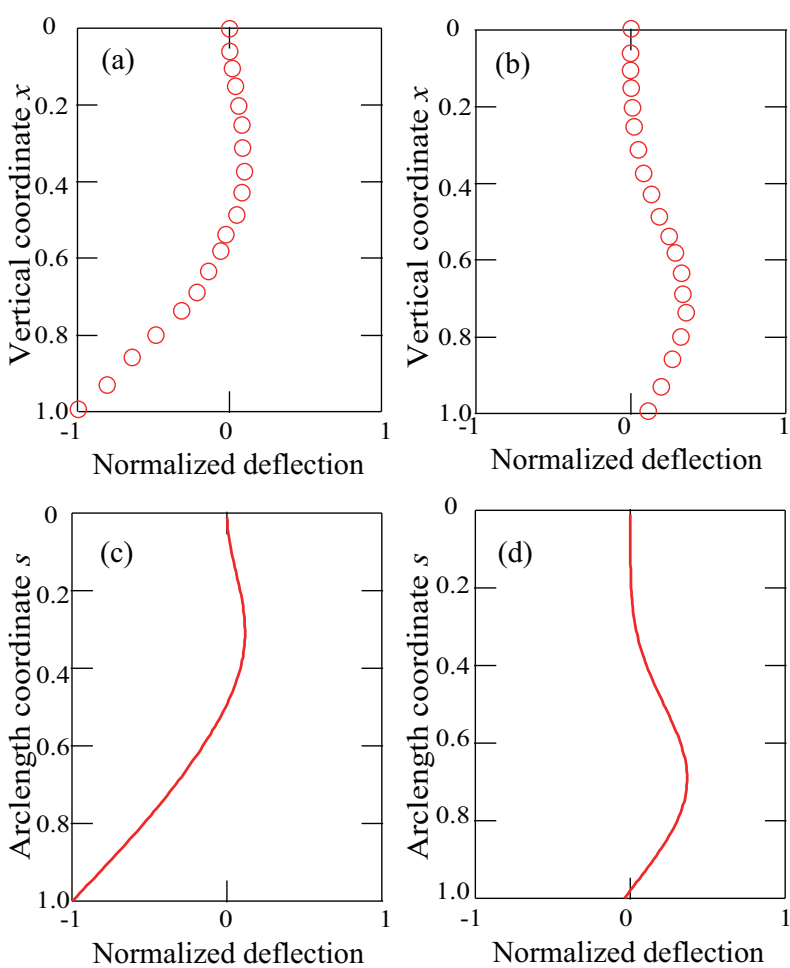

Fig. 10 Comparison of the experimental mode shape with the theoretical mode shape for Pipe 2: (a) and (b) shapes of the experimental real and imaginary modes and (c) and (d) shapes of the theoretical real and imaginary modes.

the normalized pixel resolution is 0.00769 , the normalized deflection at $0.134 \leq x \leq 0.361$ is $0.003-0.020$. In the middle of the mode shapes, there is a point at which the normalized deflection changes rapidly. The positions are $x=0.413$ in (b) and $s=0.390$ in (d), that is, the positions are similar.

Next, we show the mode shapes of Pipe 2 in Fig. 10. The experimental real and imaginary components of the mode shapes agree with the theoretical components qualitatively. That is to say, real components (i.e. (a) and (c)) have an antinode and a node at the middle of the mode shapes. The positions of the antinode and node are $x=0.373$ and 0.521 in (a) and $s=0.310$ and 0.490 in (c), that is, the positions are similar. Regarding the imaginary components, there is an antinode near the bottom of the mode shapes at $x=0.737$ in (b) and $x=0.690$ in $(\mathrm{d})$. There is a node at $x=0.100$ in (b) and there are two nodes at $s=0.100$ and 0.980 in (d). There is no node at $s=0.980$ in the experimental mode shape. As seen from (b) and (d), this absence is considered to be the effect of a slight error in the displacement of the lower markers .

At last, we show the mode shapes of Pipe3 in Fig. 11. Pipe 3 has the parameters given in Table 1 and its destabilized mode is the second mode unlike the case for 

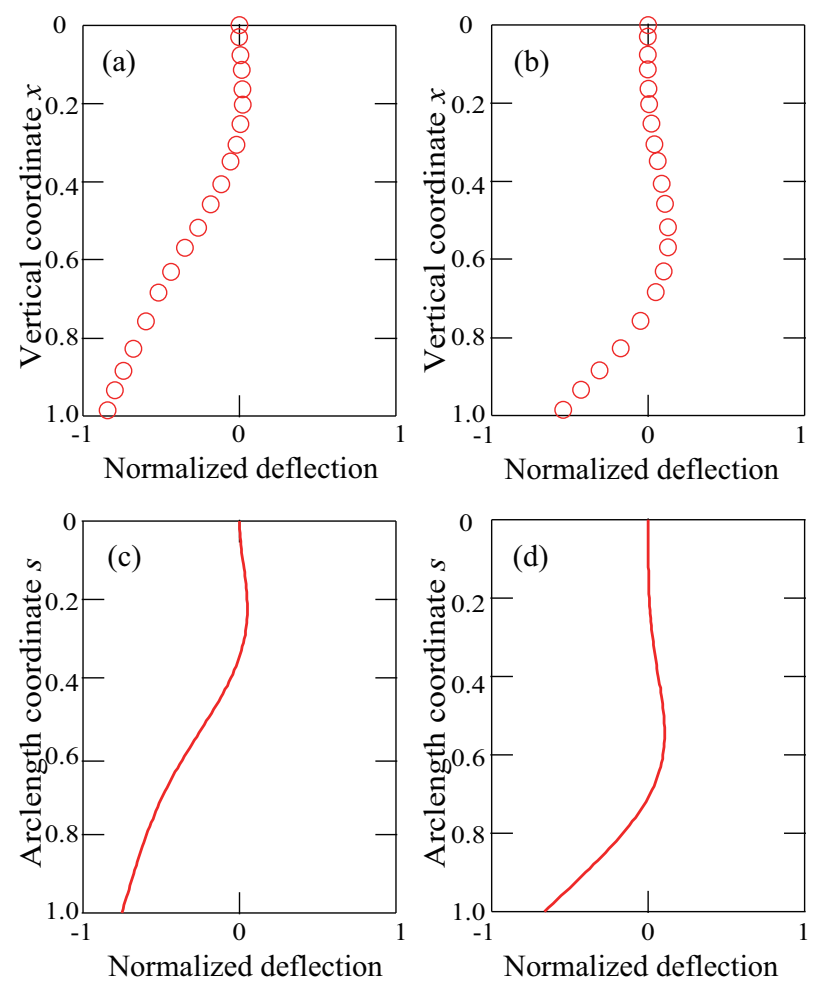

Fig. 11 Comparison of the experimental mode shape with the theoretical mode shape for Pipe 3: (a) and (b) shapes of the experimental real and imaginary modes and (c) and (d) shapes of the theoretical real and imaginary modes.

\section{Conclusion}

A flexible cantilevered pipe conveying fluid is self-excited by a nonconservative force generated by the fluid. From a mathematical point of view, the destabilization phenomenon relates to the non-selfadjointness of the governing equation, which makes the eigenmodes nonorthogonal to each other and converts the real eigenmodes to complex eigenmodes. The proposition of a method of identifying the complex modes experimentally is important to gaining a deep understanding of the self-excited vibration due to non-selfadjointness. In the present study, we first clarified theoretically the complex eigenmodes of the self-excited oscillation and investigated the effect of the nonlinearity of systems on the eigenmodes. We then proposed a method of experimentally identifying the complex mode and decomposing the mode into real and imaginary components using the time histories of some points on the pipe recorded using a high-speed video camera. For three pipes, two being self-excited with the third mode and the other with the second mode, the real and imaginary components of the selfexcited eigenmode were compared between the theoretical and experimental results. The validity of the proposed identification method was thus confirmed for the complex eigenmode of the self-excited oscillation due to the non-selfadjointness of the system.

Acknowledgements The authors thank Professor Emeritus M. Yoshizawa for useful discussions and Edanz for editing a draft of this manuscript. This work was supported by a grant from the University of Tsukuba jyu-ten-haibun.

\section{Conflict of interest}

The authors declare that they have no conflict of interest.

\section{Data availability statement}

My manuscript has no associated data.

\section{References}

1. Ashley, H., Haviland, G.: Bending vibrations of a pipe line containing flowing fluid (1950)

2. Bajaj, A., Sethna, P.: Flow induced bifurcations to threedimensional oscillatory motions in continuous tubes. SIAM Journal on Applied Mathematics 44(2), 270-286 (1984)

3. Bajaj, A., Sethna, P.t., Lundgren, T.: Hopf bifurcation phenomena in tubes carrying a fluid. SIAM Journal on Applied Mathematics 39(2), 213-230 (1980) 
Table 4 Comparison of the frequencies of free vibration of the first and second modes in the experimental results with those in the theoretical results

\begin{tabular}{lcllll}
\hline & Exp./Cal. & Pipe 1 & Pipe 2 & Pipe 3 & Unit \\
\hline Frequency (first mode) & Exp. & 1.08 & 1.08 & 1.3 & $\mathrm{~Hz}$ \\
& Cal. & 1.07 & 1.09 & 1.30 & $\mathrm{~Hz}$ \\
Frequency (second mode) & Exp. & 3.52 & 3.76 & 5.9 & $\mathrm{~Hz}$ \\
& Cal. & 3.48 & 3.67 & 5.81 & $\mathrm{~Hz}$ \\
\hline
\end{tabular}

4. Benjamin, T.B.: Dynamics of a system of articulated pipes conveying fluid-i. theory. Proceedings of the Royal Society of London. Series A. Mathematical and Physical Sciences 261(1307), 457-486 (1962)

5. Bishop, R.E.D., Fawzy, I.: Free and forced oscillation of a vertical tube containing a flowing fluid. Philosophical Transactions of the Royal Society of London. Series A, Mathematical and Physical Sciences 284(1316), 1-47 (1976)

6. Dodds, H.L., Runyan, H.L.: Effect of high-velocity fluid flow on the bending vibrations and static divergence of a simply supported pipe, vol. 2870. National Aeronautics and Space Administration (1965)

7. Ghayesh, M.H., Païdoussis, M.P., Modarres-Sadeghi, Y.: Three-dimensional dynamics of a fluid-conveying cantilevered pipe fitted with an additional spring-support and an end-mass. Journal of Sound and Vibration 330(12), 2869-2899 (2011)

8. Gregory, R., Païdoussis, M.: Unstable oscillation of tubular cantilevers conveying fluid i. theory. Proceedings of the Royal Society of London. Series A. Mathematical and Physical Sciences 293(1435), 512-527 (1966)

9. Gregory, R., Païdoussis, M.: Unstable oscillation of tubular cantilevers conveying fluid ii. experiments. Proceedings of the Royal Society of London. Series A. Mathematical and Physical Sciences 293(1435), 528-542 (1966)

10. Jendrzejczyk, J., Chen, S.: Experiments on tubes conveying fluid. Thin-Walled Structures 3(2), 109-134 (1985)

11. Jensen, J.S.: Articulated pipes conveying fluid pulsating with high frequency. Nonlinear Dynamics 19(2), 173-193 (1999)

12. Jin, J., Zou, G.: Bifurcations and chaotic motions in the autonomous system of a restrained pipe conveying fluid. Journal of Sound and Vibration 260(5), 783-805 (2003)

13. Kirillov, O.N.: Nonconservative stability problems of modern physics. de Gruyter (2013)

14. Lundgren, T., Sethna, P., Bajaj, A.: Stability boundaries for flow induced motions of tubes with an inclined terminal nozzle. Journal of Sound and Vibration 64(4), 553-571 (1979)

15. Modarres-Sadeghi, Y., Semler, C., Wadham-Gagnon, M., Païdoussis, M.: Dynamics of cantilevered pipes conveying fluid. part 3: Three-dimensional dynamics in the presence of an end-mass. Journal of Fluids and Structures 23(4), 589-603 (2007)

16. Nayfeh, A.H.: Introduction to perturbation techniques. John Wiley \& Sons (2011)

17. Païdoussis, M., Li, G.: Pipes conveying fluid: a model dynamical problem. Journal of Fluids and Structures 7(2), 137-204 (1993)

18. Paidoussis, M.P.: Fluid-structure interactions: slender structures and axial flow, vol. 1. Academic press (1998)

19. Paidoussis, M.P.: Fluid-structure interactions: slender structures and axial flow, vol. 2. Academic press (2003)
20. Sazesh, S., Shams, S.: Vibration analysis of cantilever pipe conveying fluid under distributed random excitation. Journal of Fluids and Structures 87, 84-101 (2019)

21. Seyranian, A.P., Mailybaev, A.A.: Multiparameter stability theory with mechanical applications, vol. 13. World Scientific (2003)

22. Steindl, A., Troger, H.: Nonlinear three-dimensional oscillations of elastically constrained fluid conveying viscoelastic tubes with perfect and broken o (2)-symmetry. Nonlinear Dynamics 7(2), 165-193 (1995)

23. Sugiyama, Y., Kawagoe, H., Kishi, T., Nishiyama, S.: Studies on the stability of pipes conveying fluid:(the combined effect of a spring support and a lumped mass). JSME international journal. Ser. 1, Solid mechanics, strength of materials 31(1), 20-26 (1988)

24. Thomsen, J.J.: Vibrations and stability, order and chaos (1997)

25. Wang, Y., Wang, L., Ni, Q., Dai, H., Yan, H., Luo, Y.: Non-planar responses of cantilevered pipes conveying fluid with intermediate motion constraints. Nonlinear Dynamics 93(2), 505-524 (2018)

26. Yamashita, K., Furuya, H., Yabuno, H., Yoshizawa, M. Nonplanar vibration of a vertical fluid-conveying pipe (effect of horizontal excitation at the upper end). Journal of Vibration and Acoustics 136(4) (2014)

27. Yamashita, K., Nishiyama, N., Katsura, K., Yabuno, H.: Hopf-hopf interactions in a spring-supported pipe conveying fluid. Mechanical Systems and Signal Processing 152, $107390(2021)$

28. Yamashita, K., Yagyu, T., Yabuno, H.: Nonlinear interactions between unstable oscillatory modes in a cantilevered pipe conveying fluid. Nonlinear Dynamics 98(4), 2927-2938 (2019)

29. Yoshizawa, M., Suzuki, T., Takayanagi, M., Hashimoto, K.: Nonlinear lateral vibration of a vertical fluidconveying pipe with end mass (special issue on nonlinear dynamics). JSME International Journal Series C Mechanical Systems, Machine Elements and Manufacturing 41(3), 652-661 (1998)

30. Zhang, Y., Yao, M., Zhang, W., Wen, B.: Dynamical modeling and multi-pulse chaotic dynamics of cantilevered pipe conveying pulsating fluid in parametric resonance. Aerospace Science and Technology 68, 441-453 (2017) 


\section{A Nonlinear term}

The nonlinear term in Eq. (35) is expressed as

$$
\begin{aligned}
n\left(\Phi_{n 1}\right) & =\left\{-2 i \omega_{n}^{2} \bar{\Phi}_{n 1}^{\prime} \int_{0}^{s} \Phi_{n 1}^{\prime 2} d s-i U_{n c r} \sqrt{\beta} \omega_{n} \Phi_{n 1}^{\prime 2} \bar{\Phi}_{n 1}^{\prime}\right. \\
& -\frac{1}{2} U_{n c r}^{2}\left(\Phi_{1}^{\prime 2} \bar{\Phi}_{n 1}^{\prime \prime}+2 \Phi_{n 1}^{\prime} \bar{\Phi}_{n 1}^{\prime} \Phi_{n 1}^{\prime \prime}\right) \\
& -\frac{1}{2} \omega_{n}\left(\Phi_{n 1}^{\prime 2} \bar{\Phi}_{n 1}+2 \Phi_{n 1}^{\prime} \Phi_{n 1} \bar{\Phi}_{n 1}^{\prime}\right)-\frac{9}{2} \Phi_{n 1}^{\prime \prime 2} \bar{\Phi}_{n 1}^{\prime \prime} \\
& -3\left(\Phi_{n 1}^{\prime} \Phi_{n 1}^{\prime \prime} \bar{\Phi}_{n 1}^{\prime \prime \prime}+\Phi_{n 1}^{\prime \prime} \bar{\Phi}_{n 1}^{\prime \prime} \Phi_{n 1}^{\prime \prime \prime}+\bar{\Phi}_{n 1}^{\prime} \Phi_{n 1}^{\prime \prime} \Phi_{n 1}^{\prime \prime \prime}\right) \\
& -\frac{1}{2}\left(\Phi_{n 1}^{\prime 2} \bar{\Phi}_{n 1}^{\prime \prime \prime \prime}+2 \Phi_{n 1}^{\prime \prime} \bar{\Phi}_{n 1}^{\prime} \Phi_{n 1}^{\prime \prime \prime}\right) \\
& +\frac{1}{2} \bar{\Phi}_{n 1}^{\prime \prime} \int_{s}^{1}\left(\int_{0}^{s} \Phi_{n 1}^{\prime 2} d s\right) d s+\omega_{n}^{2} \bar{\Phi}_{n 1}^{\prime \prime} \int_{s}^{1} \Phi_{n 1} \Phi_{n 1}^{\prime} d s \\
& +\omega_{n}^{2} \Phi_{n 1}^{\prime \prime} \int_{s}^{1}\left(\Phi_{n 1} \bar{\Phi}_{n 1}+\Phi_{n 1} \bar{\Phi}_{n 1}^{\prime}\right) d s \\
& -\frac{\gamma}{2}\left(\bar{\Phi}_{n 1}^{\prime \prime} \int_{s}^{1} \Phi_{n 1}^{\prime 2} d s+2 \Phi_{n 1}^{\prime \prime} \int_{s}^{1} \Phi_{n 1}^{\prime} \bar{\Phi}_{n 1}^{\prime} d s\right) \\
+ & \frac{\gamma}{2}(1-s)\left(\bar{\Phi}_{n 1}^{\prime \prime} \Phi_{n 1}^{\prime 2}+2 \Phi_{n 1} \bar{\Phi}_{n 1}^{\prime} \Phi_{n 1}^{\prime \prime}\right) \\
+ & \left.\frac{1}{2}\left(\bar{\Phi}_{n 1}^{\prime \prime} \Phi_{n 1}^{\prime \prime 2}(1)+2 \Phi_{n 1}^{\prime \prime} \Phi_{n 1}^{\prime \prime}(1) \bar{\Phi}_{n 1}^{\prime \prime}(1)\right)\right\}\left|A_{n}\right|^{2} A_{n} .
\end{aligned}
$$

\section{Identification of flexural rigidity}

We need the flexural rigidity $E I$ as a parameter when we calculate the eigenmode theoretically. In this study, we determined the flexural rigidity $E I$ of the pipe so that the natural frequency of free vibration without internal flow for the first mode obtained experimentally is equal to that obtained theoretically. Using this flexural rigidity $E I$, we find that the frequency of the second mode obtained experimentally is similar to that calculated theoretically as shown in Table 4 .

\section{B Non-selfadjointness of the system}

We introduce $\Psi_{n 1}$ as the adjoint function of $\Phi_{n 1}$, which satisfies

$$
\begin{aligned}
\int_{0}^{1}\left[\Phi_{n 1}^{\prime \prime \prime \prime}\right. & +\left\{U_{n c r}^{2}-\gamma(1-s)\right\} \Phi_{n 1}^{\prime \prime} \\
& \left.+\left(2 i \omega_{n} \sqrt{\beta} U_{n c r}+\gamma\right) \Phi_{n 1}^{\prime}-\omega^{2} \Phi_{n 1}\right] \bar{\Psi}_{n 1} d s=0 .
\end{aligned}
$$

Considering the boundary conditions of Eq. (34), we note that $\Psi_{n 1}$ is the solution to the boundary value problem

$$
\begin{aligned}
\bar{\Psi}_{n 1}^{\prime \prime \prime \prime} & +\left\{U_{n c r}^{2}-\gamma(1-s)\right\} \bar{\Psi}_{n 1}^{\prime \prime} \\
& +\left(-2 i \omega_{n} \sqrt{\beta} U_{n c r}+\gamma\right) \bar{\Psi}_{n 1}^{\prime}-\omega_{n}^{2} \bar{\Psi}_{n 1}=0
\end{aligned}
$$

$$
\left\{\begin{array}{l}
\bar{\Psi}_{n 1}(0)=0 \\
\bar{\Psi}_{n 1}^{\prime}(0)=0 \\
\bar{\Psi}_{n 1}^{\prime \prime}(1)=-U_{n c r}^{2} \bar{\Psi}_{n 1}(1) \\
\bar{\Psi}_{n 1}^{\prime \prime \prime}(1)=2 i \omega_{n} \sqrt{\beta} U_{n c r} \bar{\Psi}_{n 1}(1)-U_{n c r}^{2} \bar{\Psi}_{n 1}^{\prime}(1) .
\end{array}\right.
$$

According to Eqs. (B.2) and (B3), the differential equation satisfied by $\Psi_{n 1}$ does not correspond to Eqs. (33) and (34), which $\Phi_{n 1}$ satisfies. Therefore, $\Psi_{n 1}$ is not the eigenmode and this system has non-selfadjointness. When we assume the flow velocity to be zero in Eqs. (B.2) and (B.3), these equations correspond to Eqs. (33) and (34). Therefore, the fluid conveying in the pipe makes this system non-selfadjoint. 